\title{
Implementation of the CARDS Data Standards in Five Dutch University Medical Centres
}

\author{
$\mathrm{N}$ van der Putten ${ }^{1}$, ET van der Velde ${ }^{2}$, WA Dijk ${ }^{3}$, T Maikoe $^{4}$, R Hoekema $^{5}$ \\ ${ }^{1}$ Erasmus MC Rotterdam \\ ${ }^{2}$ Leiden University Medical Center \\ ${ }^{3}$ University Medical Center Groningen \\ ${ }^{4}$ University Medical Center Utrecht \\ ${ }^{5}$ University Medical Center Nijmegen, The Netherlands
}

\begin{abstract}
This study describes the first user experience at the cardiology departments of the University Medical Centres of Rotterdam, Leiden, Groningen, Utrecht and Nijmegen with the implementation of the CARDS data standards in their cardiology information systems. The main aim of this study was to identify problems in the use of the CARDS data standards. This study is focussed on the data standards for PCI procedures. The CARDS PCI data definitions were analyzed and were compared with the data definitions in the local information systems. On average, the majority, about $75 \%$, of all CARDS data fields (total number: 113 data fields) were already available in the local information systems. About $7 \%$ of the variables revealed definition differences. The implementation of the PCI CARDS data standards in our clinical systems revealed no significant problems.
\end{abstract}

\section{Introduction}

Systematic registration of data from clinical practice is important for clinical care, local, national and international registries and audit. Systematic registration of data requires harmonization of the data to be collected. The use of harmonized and standardized data also accelerates the development and facilitates the exchange of clinical systems. The CARDS (Cardiology Audit and Registration Data Standards) project was the first initiative for standardization of data elements for clinical cardiology in Europe [1]. This project has been developed by the Irish Department of Health and Children in partnership with the European Commission and the ESC (European Society of Cardiology) in 2004. The aim of this project is to achieve consensus on data standards (variables, definitions and coding) in Europe for 3 addressed subspecialties: PCI (Percutaneous Coronary Intervention), Clinical Electrophysiology (pacemakers,
ICD's and ablation), and acute coronary syndromes [2], [3], [4]. Three expert committees, one for each priority topics, have selected and described approximately 100 variables per module. Presently, the ESC and the national cardiology societies are in the phase of discussing the implementation and dissemination of the CARDS data standards.

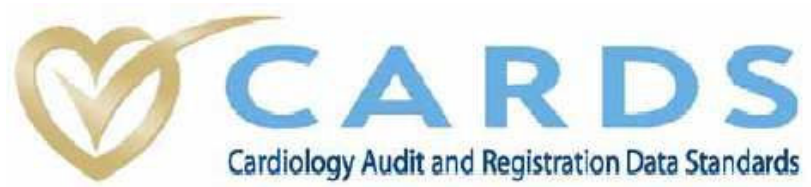

The cardiology departments of the Dutch University Medical Centers of Rotterdam, Leiden, Utrecht, Nijmegen and Groningen have started to implement the CARDS data standards in their information systems and organizations. This study is focussed on the implementation of the PCI data standards.

The following sub-objectives of this study have been formulated:

- To identify the variables that are missing from the data standards that considered to be very important to record in the Dutch PCI databases.

- To identify the variables that are present in the data standards that are considered unimportant to record in the Dutch PCI databases.

- To identify the differences in data definitions between the standard PCI data and the Dutch PCI data.

- To identify the feasibility aspects of implementing the data standards.

\section{Methods}

The data standards for PCI (and also for ACS and EP) have a consistent structure and the variables can be 
categorized under the following general headings:

- Demographics: this section contains variables such as date of birth, sex and hospital identification number.

- Past history: past medical history, previous procedures, and medication prior to hospital admission.

- Risk factors: height, weight, diabetes, hypertension, hyperlipidaemia, and current smoking status.

- Presenting symptoms: this section includes variables on presenting symptoms, clinical presentation, initial diagnosis and indication for intervention.

- Procedure: this section includes date and time of procedure, information on the type of device, the anatomical location of access and placement and medication used during the procedure.

- Outcome: this section places emphasis on both immediate and long-term complications after procedure.

- Discharge details: survival status at time of discharge, date of discharge, discharge destination and medication at discharge.

- Follow-up: this section includes survival status, events since discharge and medication at followup.

The data standards for PCI include 88 variables on the patient and the procedure, and a further 25 on follow-up.

Implementation of data standards in systems, used in clinical practice, requires a solid preparation. The implementation problems have to be assessed in a structured way.

In the first phase the overlap and the differences between the data standards and the variables and the related data definitions in the local information systems have to be investigated. In this process the following variables are identified:

1. The variables that are present in both the standards and the local information systems regardless the data definitions. A sufficient and appropriate breadth of content coverage is required!

2. The variables that are present in both the standards and the local information systems with different data definitions.

3. The variables that are present in the data standards but not available in the local information systems.

4. The variables that are present in the local information systems but not available in the data standards.

The variables in the last three sets require a very thorough examination in cooperation with local experts. The variables of set 2 are most critical. The modification of the definitions of variables may have large implications. Although a complete implementation of the card standards is desirable, some local circumstances may prevent this. Some variables are not PCI-specific. Variables such as 'risk factors for coronary artery disease' and 'left ventricular function' may belong to many different data sets. You may expect that a certain variable will have the same definitions in all data standards in due time. The conversion of the definitions of a variable in all systems requires a thorough investigation of all consequences of this operation. This process may include modification of the lay-out of paper forms, and standard reports. The relation with the historical data may be an discussion issue, too. No discussion is required if it is possible to derive the definitions of the standard data from the definitions of the local variables. The variables which are selected by a expert committee and not registered in the local information system (set 2), can be implemented without hesitation or further investigation. The variables of set 4 (locally present and not available in the standards) are probably only of local importance.

In this study we present primarily the results and experiences of this first phase of the implementation.

In the second phase of the implementation the plan for the transition from local variables and definitions into the standard data must be drawn up and realized. This includes modification of clinical and research databases, paper forms, and reports.

\section{Results}

The overlap and the differences between the CARDS data standards and the variables and the related data definitions in the local PCI systems of the University Medical Centres of Rotterdam, Leiden, Utrecht, Nijmegen and Groningen have been investigated.

\subsection{General results}

The variables in the local systems were analyzed and divided in the eight different CARDS categories (see table 1). For every category the following sets were identified:

Set1: The mean percentage of the CARDS variables that are present in the local information systems regardless the data definitions

Set2:The number of CARDS variables that are present in the local information systems with different data definitions. 


\begin{tabular}{|l|l|l|}
\hline Category & $\begin{array}{l}\text { Set 1 } \\
\text { (percentage) }\end{array}$ & $\begin{array}{l}\text { Set 2 } \\
\text { (\#variables) }\end{array}$ \\
\hline Demographics & $100 \%$ & 0 from 7 \\
\hline Past history & $75 \%$ & 0 from 8 \\
\hline Risk factors & $100 \%$ & 1 from 4 \\
\hline Presenting symptoms & $80 \%$ & 1 from 8 \\
\hline Procedure & $87 \%$ & 4 from 42 \\
\hline Outcome & $20 \%$ & 0 from 5 \\
\hline Discharge details & $64 \%$ & 1 from 14 \\
\hline Follow-up & $53 \%$ & 1 from 25 \\
\hline
\end{tabular}

Table 1: general results comparison CARDS versus local data definitions.

The percentage in Set 1 for the five individual hospitals ranged from 52 to $90 \%$ (mean value: $75 \%$ ). It is appreciated that the lowest Set 1 percentages are present in the after-procedure variables. This is mainly caused by lack of data registration systems after the PCI-procedure in some hospitals. The collection of after procedure data is often hampered by lack of information. After the PCIprocedure the patient may be transferred to another hospital without information exchange with the treating hospital.

We would expect that the percentage of correspondence between CARDS and local variables is higher if the data in other systems, such as hospital information systems, will be taken into account. Above all this is valid for the medication and discharge data.

The data in the eight different categories have been analyzed individually. The most remarkable results are discussed in subjoined sections.

\subsection{Category 'risk factors'}

In CARDS four risk factors are given in the category 'risk factors'. For some reasons the risk factor 'Family history' is not part of this list. The definition of the field 'former smoker' (patient has stopped smoking tobacco products greater than 30 days before this admission) is not in use in any local database. The definition of former smoker in the ACC-National Cardiovascular Data Registry Cath Lab Module v3.04 is also different [5].

\subsection{Category 'presenting symptoms'}

The results in category 'presenting symptoms' contain two variables in set 2 . In almost all local databases the field content of the variable 'indication for PCI' revealed more different non-ACS options than defined in CARDS. The differentiation of 'moderate left ventricular function' $(31-50 \%)$ in slightly reduced $(41-50 \%)$ and moderately reduced $(31-40 \%)$ is unknown in the Dutch local databases.

\subsection{Category 'procedure'}

The results of the comparison of the procedure variables are very good. Some CARDS variables are not present in some local databases; simply because these data are not in use in these Cathlabs. For example, if a Cathlab makes use of only one type of drug-eluting stent, the stent type is not explicitly recorded. The main differences concern the peri-procedural and percutaneous arterial complications. The CARDS field content 'Type B' of 'Type of lesion' is differentiated in 'B1' and 'B2' in most local databases.

\subsection{Rotterdam: the final implementation}

After the above mentioned comparison the university medical center of Rotterdam has implemented the CARDS PCI-definitions in their custom modified Apollo system. Only a few above discussed variables received field contents, different from the CARDS definitions. The PCI CARDS data standards has been implemented smoothly in the Apollo system within a few months. This system is operational since June 2005. The relation of the new data set with the previous data set from before June 2005, revealed no significant problems.

\section{Discussion and conclusions}

The ESC has undertaken to develop and publish clinical data standards, sets of standardized data elements and corresponding definitions that can be used in a variety of data collection efforts for a range of cardiovascular conditions. Clinical data standards are sets of standardized elements and corresponding definitions that can be used in a variety of data collection efforts. The hope of the ESC is that these standards will improve the ability to compare clinical outcomes between various trials and registries and to facilitate data management in future trials and registries. These standardized definitions also may improve quality-of-care assessment and clinical performance measures.

One important advantage of the use of data standards is underrated. The development and the exchange of cardiology information systems will be facilitated by using data standards. The software specification phase may be shorter, as the majority of the clinical data elements are already defined. The software will also less user-specific. Clinical software development is too often hampered by requirements of individual clinicians. It must be noted that not only medical data standards are required. Other important building blocks for standardized cardiology information systems are : 
- Classification systems (e.g. : ICD, SNOMED)

- Message formats (e.g.: HL7)

- System development and implementation guides

It must be noted that the greater challenge is not the development and the agreement upon a standard, but its implementation and acceptation. Implementation and acceptation are dependent factors. Both the cardiologists and the manufacturers of cardiology information systems must accept and recognize the importance of the data standards. In the ideal scenario the cardiologists require the implementation of the data standards from the vendors and manufacturers of the systems. This scenario was successfully employed by the American College of Cardiology for the implementation of the ACC PCIstandards. Broad professional agreement is required; by preference of all 47 National Cardiology Societies in Europe.

The results of this study show that there is good agreement between the CARDS data standards and the data definitions in the local information systems. The implementation of the standards in Rotterdam (78\% of the CARDS data were present in the original system) revealed an implementation without significant problems.

It must be noted that some practical problems need some attention:

- Some variables are not PCI-specific. They may belong to many different data sets. The conversion of the definitions of such a variable in all systems requires a thorough investigation of all consequences of this operation.

- The systems for the collection of some data, especially the after-procedure data, are often not cardiology-specific.

\section{References}

[1] Flynn MR, Barrett C, Cosio FG e.a. The Cardiology Audit and Registration Data Standards (CARDS), European data standards for clinical cardiology practice. Eur Heart $\mathbf{J}$ 2005;26;308-313.

[2] http://www.escardio.org/NR/rdonlyres/2D3818E7-BD5848D4-B317-A01A6AB0DBD6/0/PCIDS01104_247Kb.pdf

[3] http://www.escardio.org/NR/rdonlyres/2D5F459B-C4924E14-81A74C395D9683E3/0/EPDSFIN021104_302Kb.pdf

[4] http://www.escardio.org/NR/rdonlyres/1A88AC59-9B3A4B9D-B0E105967F985C10/0/ACSDSFin071004_180Kb.pdf

[5] http://www.accncdr.com/WebNCDR/Elements.aspx

[6] www.lumedx.com.

Address for correspondence

Niek van der Putten, $\mathrm{PhD}$

Erasmus MC

Thoraxcentre

Dr. Molewaterplein 40

3015 GD Rotterdam

The Netherlands

E-mail: n.h.j.j.vanderputten@erasmusmc.nl 